\title{
Bridging Data Management and Knowledge Discovery in the Life Sciences
}

\author{
Karl Kugler, ${ }^{* 1}$, Maria Mercedes Tejada ${ }^{2}$, Christian Baumgartner ${ }^{2}$, Bernhard Tilg $^{2}$, Armin Graber ${ }^{1}$ \\ and Bernhard Pfeifer*,2 \\ ${ }^{I}$ Institute for Bioinformatics, University for Health Sciences, Medical Informatics and Technology, Austria \\ ${ }^{2}$ Institute of Biomedical Engineering, University for Health Sciences, Medical Informatics and Technology, Austria
}

\begin{abstract}
In this work we present an application for integrating and analyzing life science data using a biomedical data warehouse system and tools developed in-house enabling knowledge discovery tasks. Knowledge discovery is known as a process where different steps have to be coupled in order to solve a specified question. In order to create such a combination of steps, a data miner using our in-house developed knowledge discovery tool $\mathrm{KD}^{3}$ is able to assemble functional objects to a data mining workflow. The generated workflows can easily be used for ulterior purposes by only adding new data and parameterizing the functional objects in the process. Workflows guide the performance of data integration and aggregation tasks, which were defined and implemented using a public available open source tool. To prove the concept of our application, intelligent query models were designed and tested for the identification of genotype-phenotype correlations in Marfan Syndrome. It could be shown that by using our application, a data miner can easily develop new knowledge discovery algorithms that may later be used to retrieve medical relevant information by clinical researchers.
\end{abstract}

\section{INTRODUCTION}

During the past years, the use of high-throughput assays and the formation of new life science related omic research fields, have led to an increase in the amount of data being produced by biomedical experiments. Until a few years ago the workbench experiment took most of the time and produced a quantity of data, which could be processed nonautomatically in most cases [1]. Nowadays, this ratio has reversed. Modern techniques like microarrays or HPLC-MS produce an enormous data output in little time, causing a need for automated data processing and integration methods. One approach for integrating and making the data harvestable is to implement a data warehouse. The following pages shall present one possible usage of data warehousing technologies in a biological research environment.

A data warehouse is a central collection or repository for persistently storing analysis relevant data and information [2-5]. Scattered and smothered under gigabytes and terabytes of data, useful information may be hidden. Data warehouses coupled with intelligent search algorithms, data mining approaches and data discovery toolboxes enable collecting and processing these data in order to turn them into useful information and new knowledge [4,6]. The conceptual design and the implementation depend, among other things, on the project or enterprise size and the used (information) systems. Therefore, the development of a data warehouse can turn out to be a tedious and costly process. These constraints make it impossible to buy a data warehouse like a standard application. The correct answer to questions like

*Address correspondence to these authors at the Institute for Bioinformatics, University for Health Sciences, Medical Informatics and Technology, Austria; E-mail: karl.kugler@umit.at

Institute of Biomedical Engineering, University for Health Sciences, Medical Informatics and Technology, Austria; E-mail: bernhard.pfeifer@umit.at what data is really key for the scientists, what is the overall impact of their data on the system, in what aggregated form and formats can the data be delivered, what data sources are available and how can they be accessed is of importance for completing a data warehouse project.

Furthermore, the amount of data in a data warehouse is usually huge due to the fact that data are stored over a longterm period to perform business intelligence (BI). An additional factor, with using biological data bases, is the exponential growth rate of information contained in public available data sources. This growth may be measured in two dimensions. One dimension represents the number of public domain data bases. The current database issue of Nucleic Acid Research counts 1078 data bases, which reflects an annual increase of about $11 \%$ in 2006 and $13 \%$ in 2007, respectively; and the other dimension being the amount of the data itself, stored in these databases [7-9].

One of the biggest challenges of building a data warehouse system is that the data warehouse administrator needs to be familiar with a lot of different "languages". One is forced to know how the various data sources can be accessed, how the different network protocols work and how the operating systems can be handled. Only if these issues are treated well, a data warehouse can be seen as useful, instead of becoming another vast silo of unusable information [10]. The different data sources are integrated and updated in periodic intervals by accessing the internal and external data sources. After consolidation the data become accessible by BI tools, which are focused on unearthing unknown facts hidden in the data. When recapitulating the above described steps, it becomes clear that a data warehouse system is not a monolithic application, it is more a complex interacting system of components, where several applications, frameworks and tools are bridged in order to accomplish these tasks. 
Data can be described as logical grouped information units and are therefore the fundamental components of information [3]. In the field of computer sciences data are understood as machine readable and processable digital representations of information. The information itself is character coded, and the information is generated using defined rules. Therefore, information follows syntax. The feasibility of interpreting data in a semantic context is indispensable for extracting information from these data.

One main objective when working with data in a data warehousing environment is to keep the data sets in the correct form, in a syntactic as well as in a semantic way. As data warehouses are tools for supporting the process of decision making, or in the actual case, the development and deployment of new biomedical knowledge, the correctness of the stored data is a crucial prerequisite [11]. Rahm and Do further point out that regarding large number of various sources for data integration, it might be a reasonable assumption, that corrupted data might be contained. They even consider cleaning this potentially corrupted data as one of the biggest challenges in data warehousing. Several manipulation and cleaning approaches are defined [11-14]. They all should be complete, meaning that all errors and inconsistencies in both the source data and the integrated data, should be found and wherever possible fixed. In an optimal case those approaches should need minimal interaction by the user, should mask the underlying data sources and should be extendable to further data sources. And last but not least, they should be combinable with schema related data transformations, enabling semantic correctness and completeness. For biological data an additional criterion is the ability to find and merge duplicated information on a semantic level. A survey done by Schönbach et al. showed that about 30\% of 145 source data contained non-critical errors, that could have caused further trouble [15].

In order to accomplish all these mentioned requirements an Extract-Transform-Load (ETL) process is introduced [2]. The ETL process may be considered as the basic concept of a data warehouse back room. It extracts the needed data from the source systems, transforms it into the needed presentation, by performing aggregations and other manipulations on the extracted data, afterwards loads these results into the data warehouse repository, and finally transforms the stored data in a user friendly representation format. By putting these steps together it is possible to state, that ETL is responsible for syntactic data integration when using the data warehouse approach. In most cases the original data is uploaded into a separate staging area, where it is transformed and possibly corrected before loading it into the final repository. To further increase data quality, either manual curation by experts or automated annotation should be performed after integration. Computational annotation has not yet been implemented but will be included in one of the next project steps. One thing that has to be kept in mind is that it is difficult to decide what data can be deleted since even incomplete or potentially incorrect data might be of interest for research purposes [1].

To prove the suitability and usefulness of our application to answer biological questions, we developed a framework for the study of correlations between genetic mutations and different phenotypic expressions. For this purpose, we used our application and a Knowledge Discovery in Databases
(KDD) approach to develop intelligent query models. Mutations in the fibrillin-1 coding gene FBN1 have been shown to cause Marfan Syndrome (MFS), a multisystemic connective tissue disorder, inherited as an autosomal dominant trait [16]. This abnormal genetic condition is characterized by pleiotropic manifestations and a high degree of clinical variability involving predominantly the ocular, skeletal, and cardiovascular systems [17]. Several studies have been focused on exploring correlations between FBN1 mutations and the clinical phenotype, as they are crucial for predicting the clinical consequences of a specific mutation [18-21]. In the absence solid correlations, the identification of an FBN1 mutation has only a little prognostic value [22].

An early and accurate diagnosis is essential for the effective treatment of MFS, before life threatening complications arise. The leading cause of death in patients with MFS is the progressive dilatation of the aorta, which can lead to dissection or rupture. The weakening of the aortic wall is one of the many consequences of abnormalities in the synthesis and secretion of fibrillin-1 caused by mutations in the FBN1 gene. This extracellular matrix glycoprotein is the major constituent of the calcium-binding microfibrils, which provide structural support in connective tissue throughout the body. Many of the clinical manifestations in MFS underlie deficiencies in the microfibrilar system [19].

\section{METHODS}

\subsection{Data Warehousing Architecture}

It is common practice to separate between back room and front room entities when talking about data warehouses. These two parts are in most cases separated physically as well as logically. While the back room is holding and managing the data, the front room enables data accession methods. This distinction is crucial in understanding how a data warehouse works and how it is organized. The back room is often described as data management or data preparation component. It contains the data, prepares and delivers data retrieved by queries, but it does not support any user queries from the outside since this is a task of the front room. A back room, in this context, may be regarded as permanently storing the information to a physical entity like a disc. In the presented application a PostgreSQL database was used as storage system (Version 8.1), further referred to as repository. For most ETL tasks the third party open source application Talend Open Studio (TOS) was used to build tasks that download data from the external data sources, transforms and cleans them, according to the manipulation necessities introduced above, and then loads them into the application's repository [23], as can be seen in Fig. (4) for a simplified workflow.

The front room component enables a user or client application to access the data held in the warehouse. The main task of the front room is mapping the huge amount of lowlevel data, usually stored in a data warehouse, to another more valuable form [24]. The front room manages the queries performed at the outside and schedules and plans them, in order to achieve the results defined for performance issues. Its activities can be referred to as Business Intelligence. The diction "intelligence" implies that non trivial actions take place in here. If defining business intelligence as "...the process of turning data into information and then into 
knowledge", as Golfarelli et al. do [25], and, at the same time, regarding the repository containing the data of the data warehouse as a database, we may set the wordings BI and $\mathrm{KDD}$ as equivalent in this context. The front room may provide techniques of data mining, text mining or classical statistical methods, being typically performed on Data Marts and multidimensional cubes.

The presented ETL framework can be regarded as consisting of two main features. One of these features is the conversion of given file formats or database formats in order to fit to the structure needed by the following loading processes, which store the data in the repository.

The right part of Fig. (1) shows the ETL-component embedded in the system environment, excluding any parts of the front room. As can be seen, data coming from external sources, may be accessed in a file representation, including simple flat files, more complex XML files or as a database, which in most cases mean, accessing and reading a data base dump. The loading component makes use of Java code and archives created by the TOS tool, which is used in order to create a simple mechanism of importing data. These files can later be re-used in the ETL component in order to read, modify and store the data. As the requirement definition for data transformation consumes about $70-80 \%$ of the time used to build a data warehouse [15], the conversion and transformation steps, including Java classes created by TOS, were the first software components to be designed and planed, in order to enable an early user interaction with establishing the warehouse.

\subsection{Bridging the Front and Back Room}

The back room component, where only the database and the data warehouse administrator has direct access, needs to be bridged with the front room. Users and scientists have access to the data via the front room for generating new knowledge and systems biology approaches without having to know database query languages like e.g. SQL. An integrated business intelligence application or toolbox is essential because it cannot be assumed that an average user is familiar with the interaction of statistical, data mining or generally knowledge discovery approaches. Furthermore, one common mistake is declaring a spreadsheet application, which can be used for basic tasks like visualization, to be the main knowledge discovery tool. To overcome the abovementioned problems a tool, named Knowledge Discovery in Databases Designer $\left(\mathrm{KD}^{3}\right)$, has been implemented as part of the presented Data Warehouse system.

To systematically study and support the discovery of novel genotype-phenotype correlations, intelligent query models were developed based on log-likelihood weights for each diagnostic criterion of MFS. The models were built using genetic and clinical data of 436 patients. Sixteen diagnostic criteria were taken into account and coded as boolean vectors. This coding is based on the fact that clinical symptoms are assumed to be either present or absent and hence they can be represented by a binary parameter. To query the database, four different levels of mutational information were defined. The models enable the assignment of a clinical phenotype of unknown mutation to a mutation class at each information level according to the type of mutation, the consequence at the protein level, the location of the mutational event, and the amino acid changes.

Two different types of queries are involved in the retrieval process: ad-hoc and intelligent queries. Ad-hoc queries are information requests that are usually constructed and run a single time. The requests are executed as SQL state-

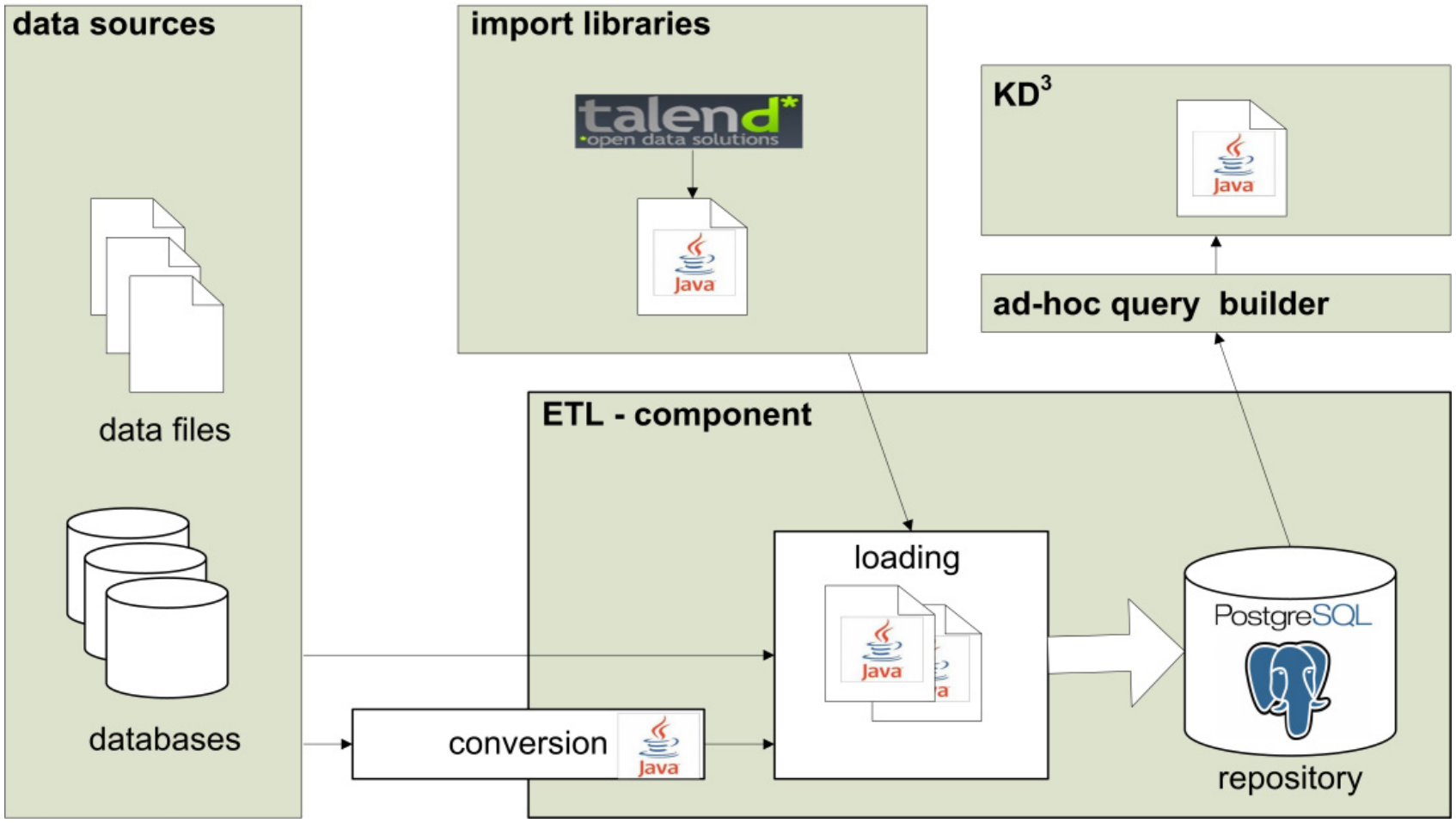

Fig. (1). The upper figure shows the main components of the presented application. The conversion and loading components for the ETL process are the main features the back room implements. As can easily be seen, the loading component contains Java source provided by Talend Open Studio. Bridging between back and front room is enabled by the ad-hoc query builder and $\mathrm{KD}^{3}$. 
ments that have been formulated by a knowledgeable user or through a query building tool. Intelligent queries are expanded queries, constructed based on the result sets of adhoc queries. These queries use additionally a medical knowledge base to process the information requests. The execution of such queries facilitates the extraction of meaningful information and the identification of patterns and relationships within the data. With the concept of different information hierarchies, queries might be answered at multiple levels of abstraction to discover implicit knowledge in the database. In other words, intelligent queries do more than just access data.

\subsection{Intelligent Query Processing}

To process a query phenotype, three query assembly substeps are carried out prior to the actual assignment to the corresponding mutation class at each information level. The workflow is depicted in Fig. (2).

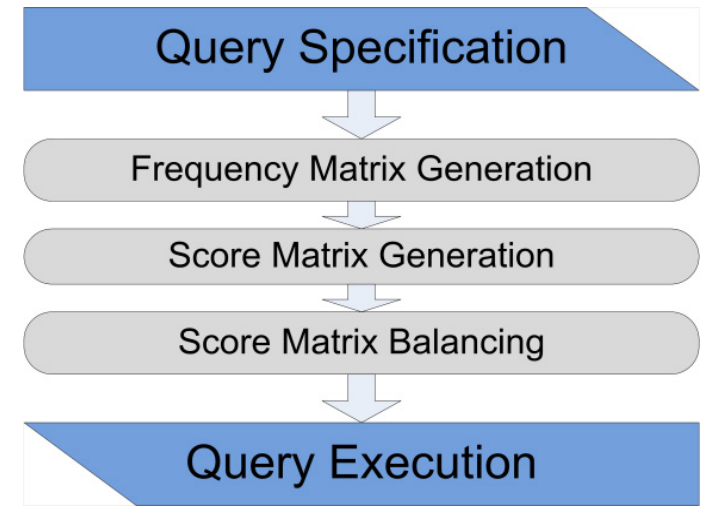

Fig. (2). Workflow intelligent query processing.

\subsubsection{Query Specification}

We define the database as a set of tuples organized in the following way

$D B=\left\{\left(c_{j}, o\right) \mid c_{j} \in C, o \in M F S \wedge o=[0,1]\right\}$

$c_{j}$ represents a specified mutation class at any of the 4 different information levels and $o$ is the set of clinical features of MFS. To assign a query phenotype given as

$O=\left\{o \mid\left(o_{1}, o 2, \ldots, o_{n}\right), o \in M F S \wedge o=[0,1]\right\}$

to a mutation class $c_{j}$, the query phenotype $O$ has to be weighted by the balanced score sets $s^{*} ; c$ for each clinical manifestation.

\subsubsection{Frequency Matrix Generation}

As a first step, we calculate the frequency of the clinical features for each mutation class $c_{j}$ and generate a frequency matrix $M_{f}$.

\subsubsection{Score Matrix Generation}

Our scoring approach is based on the relative entropy of two probability distributions, since this concept can be used to quantify the information content of a piece of data. Furthermore, it can be seen as a distance measure between the two probability distributions. The relative entropy [26] is defined as

$H(P, Q):=-\sum_{i=1}^{n} p_{i} \log _{2} \frac{p_{i}}{q_{i}}$

where $p_{i}$ is the frequency of a symptom within mutation class $c_{j}$, and $q_{i}$ is the frequency of the same symptom within the mutation classes to which class $c_{j}$ is being compared. A single score set is computed in the following way

$s_{i}=\lambda \cdot p_{i} \log _{2} \frac{p_{i}}{q_{i}}$

where $\lambda$ is a scaling factor $(\lambda=100)$. For each clinical feature a score value is computed and a matrix $M_{s}$ of score values can be generated. The single score values cannot be used as criteria to determine the similarity degree of a query phenotype and a mutation class in the database, therefore it is necessary to compute the absolute score value $s_{c}$. This parameter indicates the degree of similarity between a mutation class $c_{j}$ and the query phenotype. It is computed as

$s_{c}=\sum_{i=1}^{n}\left|s_{i}\right|$

\subsubsection{Score Matrix Balancing}

The absolute score $s_{c}$ is actually not well suited as similarity measure, since it can significantly differ in size and lead to an erroneous assignment of the query phenotype to the class with the highest $s_{c}$ within the matrix. Due to this fact, we balance the score matrix $M_{s}$ by using a correction factor $f_{c}$ given as

$f_{c}=\frac{\overline{s_{c}}}{s_{c}}$

where $\overline{s_{c}}$ is the mean absolute score value of all mutation classes. This correction factor is applied to every score set $s_{c} \in M_{s}$.

\subsubsection{Query Execution}

To assign a query phenotype to the corresponding mutation class, four decision rules were defined for each information level. These rules include the balanced score sets $s^{*} ; c$ and several weighting factors derived from reported genotype-phenotype correlations. The query phenotype $O$ is assigned to the mutation class $c_{j}$ with the highest result of the decision rule.

The decision rule at the first information level (i.e. mutation type (DNA level)) is defined as

$$
L 1:=\arg \max \left(\sqrt{\sum_{i=1}^{n}\left(s_{i}^{*} \cdot o_{i}\right)^{2}}\right)
$$


where $s^{*} ; i$ is the set of balanced score values and $o$ is the set of clinical features coded as a boolean vector.

At the second information level (i.e. mutation type and consequence (DNA and protein level)) the decision rule was defined as

$L 2:=\arg \max \left[\left(\sqrt{\sum_{i=1}^{n}\left(s_{i}{ }^{*} \cdot 0_{i}{ }^{2}\right)}\right)+\sqrt{\left(o c u \cdot \bar{S}_{o c u}\right)^{2}+\left(c s v \cdot \bar{s}_{c s v}\right)^{2}+\left(s k e \cdot \bar{s}_{s k e}\right)^{2}}\right]$

The first term of the rule is equal to the first level, but it is expanded by three weighting factors. The clinical features affecting the ocular (ocu), cardiovascular (csv) and skeletal system (ske) are weighted depending on the number of present symptoms. For this purpose, the mean score value of each organ system is computed. These values are denoted by $s ;{ }_{\text {ocu }}, s ;{ }_{c s v}^{-}$and $s ;{ }_{s k e}^{-}$respectively. The number of involved criteria is expressed by ocu, cvs, and ske.

At the third information level (i.e. mutation type, consequence, and location of the mutational event (DNA, protein and affected exon level)) the decision rule was defined in the following way

$L 3:=\arg \max \left[\left(\sqrt{\sum_{i=1}^{n}\left(s_{i}^{*} \cdot 0_{i}{ }^{2}\right)}\right)+\sqrt{\left(o c u \cdot \bar{s}_{o c u}\right)^{2}+\left(c s v \cdot \bar{s}_{c s v}\right)^{2}+\left(m \cdot \bar{a}_{a v g}\right)^{2}}\right]$

This rule is very similar to the second level, but here the patient's age is also taken into account. This is mainly because of the age related occurrence of mutations within the exons 24 to 32, the so called "neonatal region". There is no available data of genotype - phenotype correlations regarding the skeletal system. For this reason this organ system was not weighted in the decision rule.

In the fourth information level (i.e. mutation type, consequence, and amino acid changes) we applied following decision rule

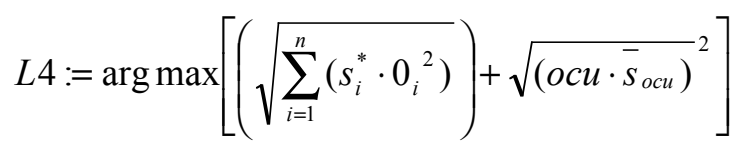

According to the reported genotype - phenotype correlations in [18], patients with mutations involving a cysteine residue present a higher risk of ectopia lentis (EL), hence higher score values are expected for this clinical manifestation. As there are no other correlations regarding the amino acid changes, only the weighting factor for the ocular system was considered.

\section{4. $\mathrm{KD}^{3}$ Front Room Application}

\subsubsection{Workflow Designer}

Many different steps are involved in a knowledge discovery approach [24]. These steps incorporate focusing and describing the problem, preprocessing and transformation of the data, performing statistical analyses and data mining algorithms, and a concluding evaluation. As data are delivered in an integrated and consistent form when using our data warehouse system, the preprocessing and transformation process can be reduced. Therefore, one can focus on building analyzing workflows for generating new knowledge. Normally more steps have to be bridged for answering a specified problem. Beside this, these steps are dependent among each other and can therefore be coupled using a workflow pipeline concept. A workflow is characterized as a repeatable pattern activity, which is used to solve a defined process using different parameters and data sets.

One idea behind $\mathrm{KD}^{3}$ is to deploy two different versions. One development version for the more sophisticated user, being a data mining specialist or a developer of biomedical algorithms, which allows creating and implementing specialized junks of code that perform some of the data manipulation tasks introduced above. A second version, referred to as deployment version allows the execution of predefined and deployed workflows. This deployment version focuses especially on clinicians, who are interested in a simple to use application, which computes the desired result. Normally, these researchers are not interested in designing or changing a given workflow or data mining approach, but are rather looking for an application that enables them to develop new findings. Fig. (3) depicts a screenshot of KD [3].

The main window of the application can be divided into four parts. In the most left window the available functional objects or tasks are displayed using a hierarchical structure. The functional objects are classes derived from the abstract superclass KDDTask, which are loaded using the Java reflection API. The advanced Java feature reflection can be used by applications, which require the ability to examine or modify the runtime behavior of applications. Thus, the $\mathrm{KD}^{3}$ application enables to make use of external, user-defined classes by creating instances of extensibility objects. Using this technique enables different users to extend the $\mathrm{KD}^{3}$ by their own algorithmic approaches without having to know anything about application internals. Only the composition of the abstract KDDTask class is necessary for enabling software developers to write plug-ins.

The light gray window is for designing the workflow. The user can drop functional objects and parameterize them by setting up the constructor. This constructor is the main function that is called by the system during instantiating the functional object. It is used for initializing the object with default parameters or for iterating through the objects result set. On the right side of the $\mathrm{KD}^{3}$ application main window the designed workflow is visualized using GraphML [27]. The GraphML representation is used for storing and deploying the workflow. The right lower window section is used for displaying intermediate results, logging, or debug information and is used by the data mining specialist for designing problem solvers.

Functional Objects are the key elements of the application. They are composed as following: From the users perspective a task is a functional object, which consists of inand out-ports. Functional objects have to be assembled and parameterized in order to fulfill their purpose (e.g. computing, querying a database, interacting with other objects). From a software engineer's point of view a functional object must be derived from the super class named KDDTask as mentioned above. A defined functional object consists of a description field, where information how to configure the constructor is given to the user. Furthermore, the in-ports have the job to retrieve data from another object, process 


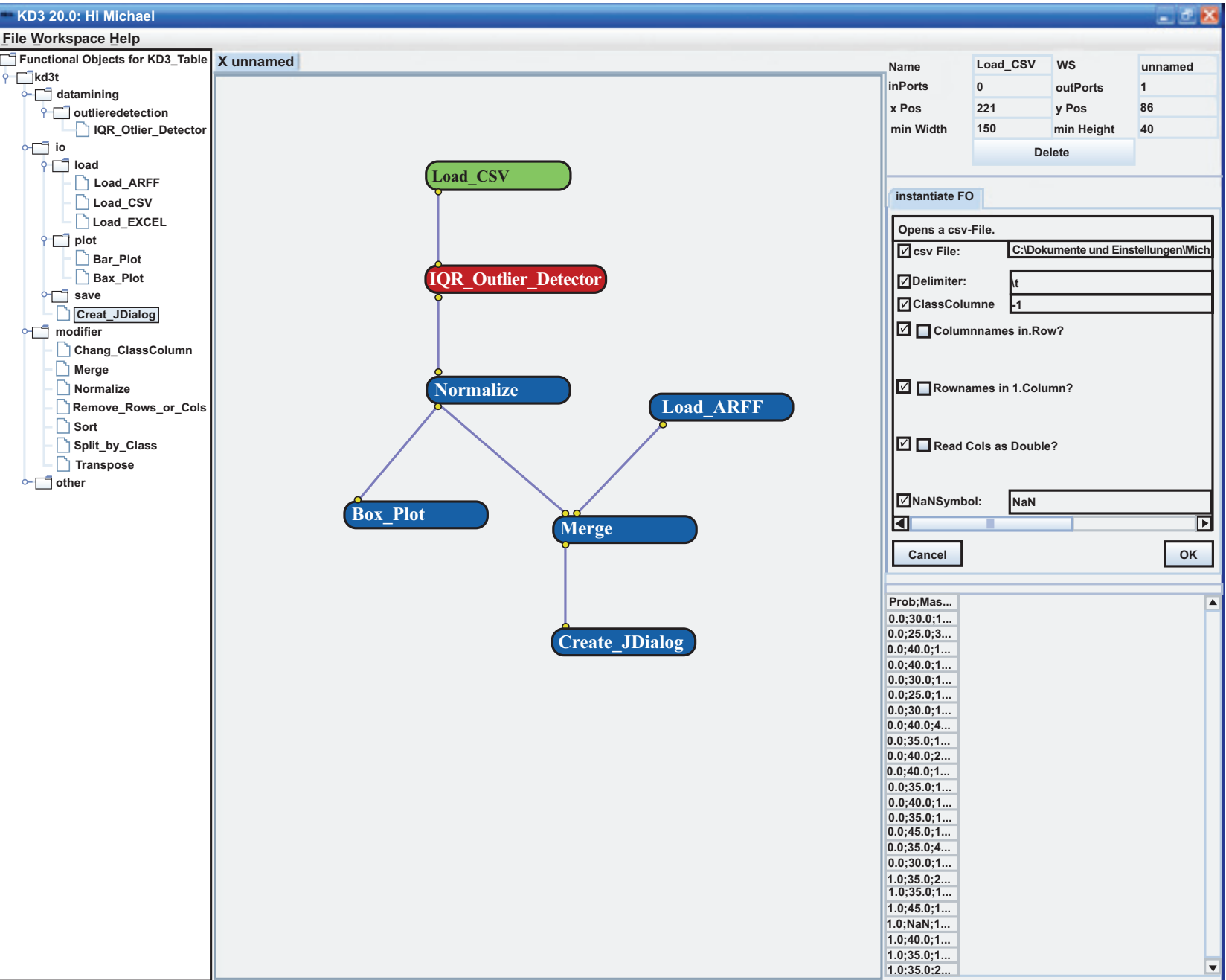

Fig. (3). Screenshot of the $\mathrm{KD}^{3}$ application. The shown workflow was implemented for data mining purposes.

those data, and send these data to another object using outports. The computation is done by calling the compute 0 method, which is abstract in the super class. This method must be overridden by implemented plug-ins.

\section{RESULTS}

The usage of TOS for designing and semiautomatically implementing ETL tasks in Java, enables a fast way of adapting to new data sources. ETL tasks can conceptually be split into atomic actions, which can be assembled in order to carry out specific functions. TOS refers to this functions as jobs, being composed by using a graphical design tool, generating Java code, that performs the specified commands. Those jobs can be used as the needed ETL tasks. It is even possible to program in the large, by combing several jobs in order to model a workflow.

The presented $\mathrm{KD}^{3}$ application enables data mining experts to design bioinformatic approaches using a graphical interface, and furthermore, non-data-base and data mining experts are enabled to study the results without having to know about technical details. The possibility to integrate custom made classes and external frameworks makes the application framework highly adaptable and expandable. Furthermore, the $\mathrm{KD}^{3}$ application is created to bridge the back room with the front room component in an easy and understandable way in order to become the main BI tool. The described and implemented MFS phenotype-genotype correlation mining workflow is a good example of what BI was meant to deliver and which possibilities the presented application has.

Table 1 shows the score matrix containing the score sets for each clinical feature. The entries represent phenotype scores based on log-likelihood weights, calculated according to equation 4 . Since the score sets are generated based on the frequencies of the symptoms, the values show a similar tendency. Regarding a single clinical feature, negative score values imply a lower frequency, when two or more mutation classes are compared. For example, EL has a frequency of $64 \%$ within the substitutions and only $37 \%$ within the NonSubstitutions. Consequently, the score values for EL constitute 50 and -29 respectively. The accumulated absolute score $s_{c}$ (eq. 5) can be used to determine the similarity degree between a query phenotype and the database. The larger this parameter, the lower the degree of phenotypic similarity. Since the $s_{c}$ values significantly vary in size, it can be deduced that a query phenotype could be wrongly assigned to certain mutation classes with the highest absolote scores 


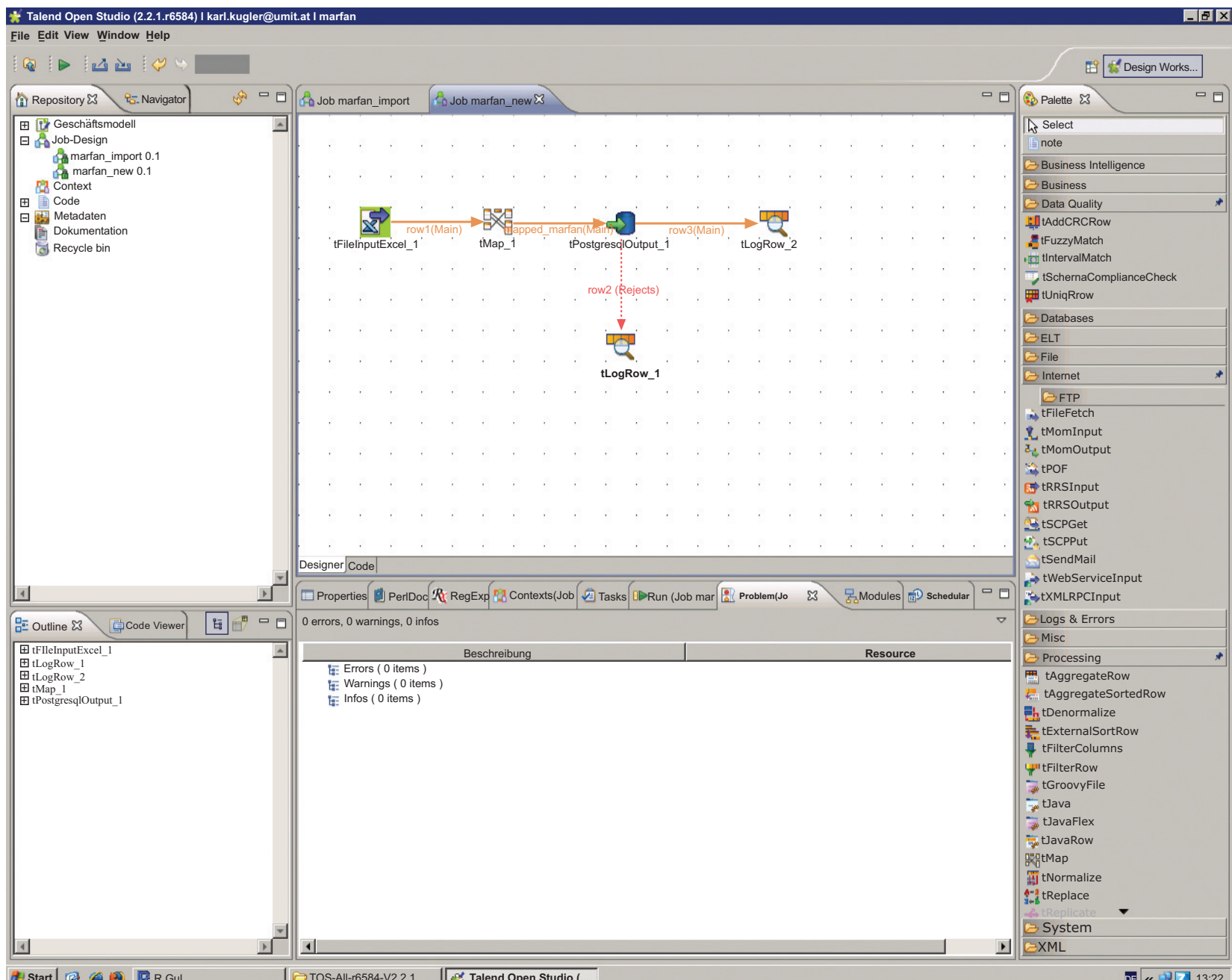

Fig. (4). TOS enables to combine several atomic actions and jobs in order to represent a certain ETL workflow. The upper screenshot shows a workflow for a data integration project.

Table 1. Score Values for Each Clinical Manifestation at the Different Levels of Mutational Information

\begin{tabular}{|c|c|c|c|c|c|c|c|c|c|c|c|c|c|c|c|c|c|c|}
\hline Level & Class & EL & MY & AADIL & AADIS & MVP & PC & PES & ASR & SC & $\mathbf{P E}$ & JH & HAP & ARA & DOL & SA & HE & sc \\
\hline \multirow[t]{2}{*}{ L1 } & Sub: & 50 & -6 & -5 & -1 & -12 & 3 & 0 & 0 & -8 & -10 & -17 & -11 & 12 & -4 & -26 & -10 & 175 \\
\hline & Mis: & 36 & -3 & -6 & -2 & 8 & -5 & 0 & -6 & -5 & -7 & -5 & -13 & 2 & -6 & -17 & -3 & 124 \\
\hline \multirow[t]{4}{*}{ L2 } & Stop: & -11 & 2 & 32 & 10 & -17 & 18 & 4 & -1 & 10 & 1 & -14 & 37 & 1 & 10 & 28 & -4 & 200 \\
\hline & Splice: & 5 & 1 & -24 & -6 & -9 & -1 & 0 & 13 & -12 & -1 & 11 & -12 & 12 & 1 & -19 & -7 & 134 \\
\hline & Mis e24-32: & -24 & 1 & 4 & -3 & 36 & 9 & 1 & -5 & 1 & -3 & 17 & -2 & 7 & -1 & -14 & 14 & 142 \\
\hline & Mis otherEx: & 34 & -1 & -4 & 3 & -24 & -7 & -1 & 5 & -1 & 3 & -14 & 2 & -7 & 1 & 23 & -8 & 138 \\
\hline \multirow[t]{3}{*}{ L3 } & Stop e24-32: & -23 & -9 & -6 & 0 & 67 & -3 & 0 & 0 & -28 & 11 & -9 & 0 & 45 & 0 & 0 & 0 & 201 \\
\hline & Mis Cys: & 65 & 7 & -9 & 3 & 13 & 19 & 0 & 1 & 1 & 0 & 4 & 11 & 8 & 11 & 17 & 5 & 174 \\
\hline & Mis otherAA: & -37 & -6 & 10 & -3 & -11 & -12 & 0 & -1 & -1 & 0 & -4 & -9 & -7 & -8 & -12 & -4 & 125 \\
\hline
\end{tabular}


(Table 1). Contd.....

\begin{tabular}{|c|c|c|c|c|c|c|c|c|c|c|c|c|c|c|c|c|c|c|}
\hline Level & Class & EL & MY & AADIL & AADIS & MVP & $\mathbf{P C}$ & PES & ASR & SC & PE & $\mathbf{J H}$ & HAP & $\mathbf{A R A}$ & DOL & SA & HE & sc \\
\hline \multirow[t]{4}{*}{ L4 } & Stop Cys: & 0 & 55 & -6 & 0 & 67 & 0 & 0 & 0 & 85 & 96 & 149 & 0 & 45 & 0 & 30 & 77 & 610 \\
\hline & Stop otherAA: & 0 & -31 & 7 & 0 & -33 & 0 & 0 & 0 & -47 & -35 & -53 & 0 & -33 & 0 & -22 & -15 & 276 \\
\hline & Fs Cys: & -14 & -23 & 43 & 6 & -25 & 15 & 0 & 11 & 13 & -3 & 13 & -7 & 1 & -17 & -16 & -9 & 216 \\
\hline & Fs otherAA: & 26 & 60 & -30 & -4 & 43 & -11 & 0 & -9 & -11 & 4 & -11 & 7 & -1 & 35 & 20 & 14 & 286 \\
\hline
\end{tabular}

preferably. The highest $s_{c}$ values were calculated for the class of nonsense mutations involving a cysteine (610) followed by the frameshift mutations within exons 24-32 (357). To avoid the erroneous assignment of the query phenotype to the class with the highest $s_{c}$ we generate a balanced score matrix. The balancing is accomplished by computing a correcting factor (eq. 6) and applying it to all score sets in the matrix. The resulting balanced matrix (not explicitly shown) is used for the actual phenotype assignment to the corresponding mutation class at each information level.

\section{DISCUSSION \& CONCLUSION}

The front to back room bridging application $\mathrm{KD}^{3}$ enables the data miner or BI specialist to predefine deployable workflows in order to turn data into information to gain new knowledge. Apart from this, the finding of unknown patterns and decision support or helping to enhance medical treatment is also targeted. The developed and tested MFS workflow can be deployed and used by other researchers and medical staff using such a distributable version. Furthermore, external frameworks and toolboxes can be integrated by implementing an adaptor class. For use with other Data Mining methods, we integrated the well-known data mining framework WEKA [28] into our $\mathrm{KD}^{3}$ designer tool. Motivated by biomedical research projects an easy to use and easy expandable system has been developed. The presented application tries to bridge the gap between the back and front room component in a biomedical data warehouse project.

All mentioned software tools are accessible on request to the authors.

\section{ACKNOWLEDGEMENTS}

We want to thank Andreas Dander and Michael Handler for their contribution to the $\mathrm{KD}^{3}$ project, Gerd Lorünser and Robert Rostek for developing the ad-hoc query builder and Martin Lange and Yves de Montcheuil from Talend.

\section{REFERENCES}

[1] T. Hernandez and S. Kambhampati, "Integration of biological sources: current systems and challenges ahead", SIGMOD Rec., vol. 33, no. 3, pp. 51-60, September 2004.

[2] R. Kimball and J. Caserta, The data warehouse ETL toolkit. Wiley Publishing, Indianapolis: 2000.

[3] R. Kimball and M. Ross, The data warehouse toolkit: The complete guide to dimensional modeling (Second Edition). Wiley Publishing, Indianapolis: 2002.

[4] A. Bauer and H. Günzel, data warehouse systeme. Heidelberg: dpunkt.verlag, 2004.

[5] G. Allen, Ed., Data warehousing tools and solutions. Towcester: Bloor Research Group, 1997.

[6] B. Pfeifer, J. Aschaber, C. Christian, S. Dreiseitl, R. ModreOsprian, G. Schreier, and B. Tilg, "A data warehouse for prostate cancer biomarker discovery", in BIOCOMP 2007: International conference on bioinformatics \& computational biology, 2007, pp. 316-323.

[7] M. Y. Galperin, "The molecular biology database collection: 2008 update", Nucleic Acids Res., vol. 36, no. "Database-Issue", pp. 2-4, November 2007.

[8] M. Y. Galperin, "The molecular biology database collection: 2007 update," Nucleic Acids Res., vol. 35, no. "Database-Issue", pp. 3-4, January 2007.

[9] Swiss Institute of Bioinformatics, "UniProtKB/Swiss-Prot protein knowledgebase release 54.8 statistics", 2008.

[10] S. P. Gardner, "Ontologies and semantic data integration," Drug Discov. Today, vol. 10, no. 14, pp. 1001-1007, July 2005.

[11] E. Rahm and H. H. Do, "Data cleaning: problems and current approaches", IEEE Data Eng. Bull., vol. 23, no. 4, pp. 3-13, September 2000 .

[12] U. Leser and P. Rieger, "Integration molekularbiologischer daten", Datenbank-Spektrum, vol. 6, pp. 56-66, June 2003.

[13] L. M. Haas, E. T. Lin, and M. A. Roth, "Data integration through database federation”, IBM Syst. J., vol. 41, no. 4, pp. 578-596, October 2002.

[14] I. K. Ibrahim and W. Schwinger, "Data integration in digital libraries: approaches and challenges", in IDLN 2001: Proceedings of the International Seminar on Digital Library and Knowledge Management, June 2001.

[15] C. Schönbach, P. Kowalski-Saunders, and V. Brusic, "Data warehousing in molecular biology", Brief. Bioinform., vol. 1, no. 1, pp. 190-198, May 2000.

[16] D. Halliday, S. Hutchinson, L. Lonie, J. Hurst, H. Firth, P. Handford, and P. Wordsworth, "Twelve novel FBN1 mutations in marfan syndrome and marfan related phenotypes test the feasibility of FBN1 mutation testing in clinical practice", J. Med. Genet., vol. 39, no. 8, pp. 589-593, December 2002.

[17] P. Comeglio, P. Johnson, G. Arno, G. Brice, A. Evans, J. AragonMartin, F. P. da Silva, A. Kiotsekoglou, and A. Child, "The importance of mutation detection in marfan syndrome and marfan-related disorders: Report of 193 FBN1 Mutations", Hum. Mutat., vol. 28, no. 9 , p. 928 , September 2007

[18] L. Faivre, G. Collod-Beroudand, B. Loeys, A. Child, C. Binquet, E. Gautier, B. Callewaert, E. Arbustini, K. Mayer, M. ArslanKirchner, A. Kiotsekoglou, P. C. glio, N. Marziliano, H. Dietz, D. Halliday, C. Beroud, C. Bonithon-Kopp, M. Claustres, C. Muti, H. Plauchu, P. N. Robinson, L. C. Adès, A. Biggin, B. Benetts, M. Brett, K. J. Holman, J. D. Backer, P. Coucke, U. Francke, A. D. Paepe, G. Jondeau, and C. Boileau, "Effect of Mutation type and location on clinical outcome in 1,013 Probands with marfan syndrome or related phenotypes and FBN1 mutations: an international study", Am. J. Hum. Genet., vol. 81, pp. 454-466, September 2007.

[19] P. Robinson and M. Godfrey, "The molecular genetics of Marfan syndrome and related microfibrillopathies", J. Med. Genet., vol. 37, no. 1, pp. 9-25, January 2000.

[20] I. Schrijver, W. Liu, T. Brenn, H. Furthmayr, and U. Francke, "Cysteine substitutions in epidermal growth factor-like domains of fibrillin-1: distinct effects on biochemical and clinical phenotypes", Am. J. Hum. Genet., vol. 65, no. 4, pp. 1007-20, October 1999.

[21] F. Tiecke, S. Katzke, P. Booms, P. Robinson, L. Neumann, M. Godfrey, K. Mathews, M. Scheuner, G. Hinkel, R. Brenner, H. Hovels-Gurich, C. Hagemeier, J. Fuchs, F. Skovby, and T. Rosenberg, "Classic, atypically severe and neonatal Marfan syndrome: twelve mutations and genotype-phenotype correlations in FBN1 exons 24-40", Eur. J. Hum. Genet., vol. 9, pp. 13-21, January 2001.

[22] C. Baumgartner, G. Mátyás, B. Steinmann, M. Eberle, J. Stein, and D. Baumgartner, "A bioinformatics framework for genotype- 
phenotype correlation in humans with Marfan syndrome caused by FBN1 gene mutations", J. Biomed. Inf., vol. 39, no. 2, pp. 171-183, April 2006.

[23] Talend Open Studio, [Online]. Available: http://www.talend.com [Accessed March 23, 2007].

[24] U. M. Fayyad, G. Piatetsky-Shapiro, and P. Smyth, "From data mining to knowledge discovery: An overview", in Advances in Knowledge Discovery and Data Mining, U. M. Fayyad, G. Piatetsky-Shapiro, P. Smyth , and R. Uthurusamy, Ed. Menlo Park: AAAI Press, 1996, pp. 1-34.

[25] M. Golfarelli, S. Rizzi, and I. Cella, "Beyond Data warehousing: what's next in business intelligence?" in DOLAP 2004: Proceed- ings of the 7th ACM international workshop on data warehousing and OLAP, November 2004, pp. 1-6.

[26] S. Kullback and R. A. Leibler, "On information and sufficiency", Ann Math Statist, vol. 22, no. 1, pp. 79-86, March 1951.

[27] GraphML, [Online]. Available: http://graphml.graphdrawing.org [Accessed Feb. 01, 2007].

[28] I. H. Witten and E. Frank, Data Mining: Practical machine learning tools and techniques, Second Edition. San Francisco: Morgan Kaufmann, 2005.

(C) Kugler et al.; Licensee Bentham Open.

This is an open access article distributed under the terms of the Creative Commons Attribution License (http://creativecommons.org/licenses/by/2.5/), which permits unrestrictive use, distribution, and reproduction in any medium, provided the original work is properly cited. 